\title{
STATISTICAL ANALYSIS OF THE 2xN V-BLAST ALGORITHM OVER RAYLEIGH FADING CHANNEL
}

\author{
Sergey Loyka \\ School of Information Technology and Engineering (SITE) \\ University of Ottawa, 161 Louis Pasteur, Ottawa, Ontario, Canada, K1N 6N5 \\ (e-mail: sergey.loyka@ieee.org)
}

\begin{abstract}
V-BLAST algorithm is an attractive simple solution for the Rx processing of a MIMO system. This paper presents an analytical analysis of some aspects of the algorithm performance over a flat-fading Rayleigh channel. Closed-form rigorous analytical expression for the joint outage probability and its PDF at the $1^{\text {st }}$ detection step are derived for the case of $2 \times n$ system. Corresponding distribution moments are also evaluated, and asymptotic expressions are given. The analytical results are validated through extensive Monte-Carlo simulations.
\end{abstract}

Index Terms-MIMO, V-BLAST, multi-antenna system, fading, BER, outage

\section{INTRODUCTION}

Multi-antenna (MIMO) systems attract significant attention during the last few years due to an extraordinary high spectral efficiency they promise. A key part of the system is the receiver $(\mathrm{Rx})$ signal processing algorithm. The first proposed algorithms were the D- and V-BLAST [1-3]. While the D-BLAST achieves the full MIMO capacity, it is more complex as compared to the VBLAST, which, despite its simplicity, achieves a significant portion of the full MIMO capacity. Despite their popularity, their performance is not understood well yet (especially when compared to traditional digital communication systems, whose performance is understood quite well by now). Mostly, the algorithms were analyzed using numerical techniques (i.e., MonteCarlo simulations). While this approach is able to predict the performance (i.e., BER, outage probability, etc.) quite accurately for any specific scenario, it lacks a deep insight usually provided by analytical techniques. Due to their complexity, the V and D-BLAST algorithms present a serious difficulty for an analytical analysis.

In this paper, we follow the traditional approach to the performance analysis of wireless communication systems over fading channels $[4,5]$. The two key parameters are the outage probability (i.e., the probability that the instantaneous SNR exceeds given threshold level) and the average BER (averaged over the channel statistics). The latter is expressed as:

$$
\overline{P_{e}}=\int_{0}^{\infty} \rho(\gamma) P_{e}(\gamma) d \gamma
$$

where $\overline{P_{e}}$ is the average BER, $P_{e}(\gamma)$ is the instantaneous BER (i.e. for given instantaneous SNR $\gamma$ ), and $\rho(\gamma)$ is the probability density function (PDF) of $\gamma$. The outage probability can be expressed as

$$
P_{\text {out }}\left(\gamma_{t h}\right)=\int_{0}^{\gamma_{t h}} \rho(\gamma) d \gamma=F_{\gamma}\left(\gamma_{t h}\right)
$$

i.e. it is given by the cumulative distribution function (CDF) of $\gamma$.

In the context of multi-antenna (MIMO) systems, the approach has to be extended to account for multiple bit streams, which are lunched by the $\mathrm{Tx}$ antennas simultaneously. This results in multivariate PDF and CDF. The key parameters are still the average BER and the outage probability, which are, however, different at different detection steps (i.e., for different bit streams). We employ the method developed in $[6,7]$ and analyze analytically a $2 x n$ V-BLAST (i.e., 2 Tx and $n$ Rx antenna system). In particular, we derive the joint (multivariate) PDF and CDF of the after-processing signal powers at the $1^{\text {st }}$ detection step. The latter is, in fact, the outage probability, and the former is critical for the average BER analysis, as (1) indicates. Note that the SNR is proportional to the signal power and, hence, has the same density (distribution) function when normalized properly. While the analysis in this paper is limited to the $1^{\text {st }}$ step only, we note that (i) it can also be extended to the $2^{\text {nd }}$ step, and (ii) at the high SNR mode the total BER is dominated by the $1^{\text {st }}$ step BER and, hence, it is crucial to understand well the latter.

The analysis given in the paper results in closed-form rigorous analytical expressions, which are quite simple for low-order systems, i.e. for $2 \times 2$ and $2 \times 3$ ones. Asymptotic behavior of the outage probability is discussed as well. It is shown that these functions facilitate the analysis of the optimal ordering procedure and provide a significant insight in its performance. Finally, the analytical results are verified by extensive Monte-Carlo simulations.

\section{V-BLAST ALGORITHM}

For completeness, we outline here the major steps of the V-BLAST algorithm (for details, see for example [3]). The main idea of the BLAST architecture is to split the information bit stream into several sub-streams and transmit them in parallel using a set of Tx antennas (the number of $\mathrm{Tx}$ antennas equals the number of substreams) at the same time and frequency. At the Rx side, each Rx antennas "sees" all the transmitted signals, which are mixed due to the nature of the wireless propagation 
channel. Using appropriate signal processing at the $\mathrm{Rx}$ side, these signals can be unmixed so that the matrix wireless channel is transformed into a set of virtual parallel independent channels (provided that the multipath is rich enough).

The standard baseband system model is used,

$$
\mathbf{y}=\mathbf{H s}+\xi
$$

where $\mathbf{s}$ and $\mathbf{y}$ are the $\mathrm{Tx}$ and $\mathrm{Rx}$ vectors correspondingly, $\mathbf{H}$ is the $2 \mathrm{xn}$ channel matrix (i.e. the matrix of the complex channel gains between each $\mathrm{Tx}$ and each $\mathrm{Rx}$ antenna), $\mathrm{n}$ is the number of $\mathrm{Rx}$ antennas, and $\xi$ is the additive white Gaussian noise (AWGN), which is assumed to be $\mathcal{C N}\left(0, \sigma_{0}^{2} \mathbf{I}\right)$, i.e. independent and identically distributed (i.i.d.) in each branch.

The job of the V-BLAST algorithm is to find $\mathbf{s}$ given $\mathbf{y}$ and $\mathbf{H}$ in a computationally-efficient way. The VBLAST processing begins with the $1^{\text {st }}$ Tx symbol and proceeds in sequence to the $\mathrm{m}$-th symbol ( $\mathrm{m}$ is the number of Tx antennas; $m=2$ in our case). When the optimal ordering procedure is employed, the Tx indexing is changed prior to the processing. The main steps of the algorithm are as follows $[1,3]$ :

1. The interference cancellation step: at the i-th processing step (i.e., when the signal from the i-th transmitter is detected) the interference from the first $i-1$ transmitters can be subtracted based on the estimations of the Tx symbols and the knowledge of the channel matrix H,

$$
\mathbf{y}_{i}^{\prime}=\mathbf{y}-\sum_{j=1}^{i-1} \mathbf{h}_{j} \hat{s}_{j}
$$

where $\mathbf{h}_{j}$ is the $\mathrm{j}$-th column of $\mathbf{H}$, and $\hat{s}_{j}$ are the detected symbols (which are assumed to be error-free).

2. The interference nulling step: based on the knowledge of the channel matrix, the interference from yet-to-be-detected symbols can be nulled out using the Gramm-Schmidt orthogonalization process (applied to the column vectors of $\mathbf{H}$ ),

$$
\mathbf{y}_{i}^{\prime \prime}=\left(\mathbf{I}-\mathbf{C}_{i+1}\right) \mathbf{y}_{i}^{\prime}
$$

where $\mathbf{C}_{i+1}$ is the projection matrix on the sub-space spanned by $\left\{\mathbf{h}_{i+1} \ldots \mathbf{h}_{m}\right\}$.

3. The optimal ordering procedure: the order of symbol processing is organized according to their afterprocessing SNRs in the decreasing order (i.e., the symbol with highest SNR is detected first).

\section{ANALYSIS OF THE V-BLAST ALGORITHM}

The following basic assumptions are employed in the present paper:

(1) The channel is random, quasistatic (i.e. fixed for every frame of information bits but varying from frame to frame), frequency independent (i.e., negligible delay spread); the components of $\mathrm{H}$ are $\mathcal{C N}(0, I)$ (i.e., i.i.d. Rayleigh fading with unit average power gain). (2) Equalpower constellation is used. (3) The Tx signals, noise and channel gains are independent of each other. (4) Perfect channel knowledge is assumed to be available at the receiver. (5) There is no performance degradation due to synchronization and timing errors.

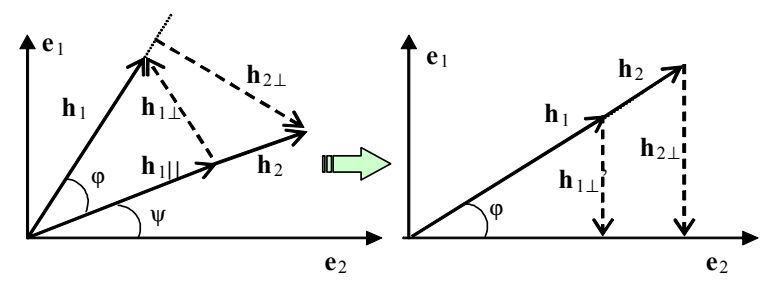

Figure 1. Geometrical representation of interference nulling out: decomposition of $h_{1}$ into $h_{1 \perp}$ and $h_{1 \|}$. $e_{1}$ and $\mathbf{e}_{2}$ are basis vectors of the space spanned by the columns of $H\left(h_{1}\right.$ and $\mathbf{h}_{2}$ ).

It should be noted that the proposed technique is flexible enough so that some of these assumptions can be relaxed, resulting, however, in a more complex analysis.

Geometrical framework for the closed-form analysis of the algorithm operation has been already discussed in details in [6,7]. Here, we summarize the major results that are used below. For the case of $\mathrm{m}=2$, the interference nulling step is illustrated in Fig. 1. Since the Rx signal coming from $\mathrm{j}$-th $\mathrm{Tx}$ is $\mathbf{h}_{j} s_{j}$ and its power is $\left|\mathbf{h}_{j} s_{j}\right|^{2}=\left|\mathbf{h}_{j}\right|^{2}$ (recall that we assume an equal power constellation), the after-processing signal power, and, hence, the SNR, at the 1st processing step is proportional to

$$
s=\max \left[\left|\mathbf{h}_{1 \perp}\right|^{2},\left|\mathbf{h}_{2 \perp}\right|^{2}\right]=(\sin \varphi)^{2} \max \left[\left|\mathbf{h}_{1}\right|^{2},\left|\mathbf{h}_{2}\right|^{2}\right]
$$

where the max is due to the optimal ordering procedure, and the orthogonal vector components are due to the interference nulling out. Note that $\left|\mathbf{h}_{1}\right|^{2},\left|\mathbf{h}_{2}\right|^{2} \sim \chi_{2 n}^{2}$, where $\sim$ means in distribution, and, as it was shown in [6,7], $\quad\left|\mathbf{h}_{1 \perp}\right|^{2},\left|\mathbf{h}_{1 \perp}\right|^{2} \sim \chi_{2(n-1)}^{2}$. The distribution $P(x)=\operatorname{Pr}[s<x]$ of $\mathrm{s}$ can be presented in the following form $[6,7]$ :

$$
P(x)=\int_{0}^{\pi / 2} F_{n}^{2}\left(\frac{x}{\sin ^{2} \varphi}\right) f_{\varphi}(\varphi) d \varphi
$$

where $F_{n}(x)=\operatorname{Pr}\left[\left|\mathbf{h}_{1}\right|^{2}<x\right]=1-e^{-x} \sum_{k=0}^{n-1} \frac{x^{k}}{k !}$ is n-th order MRC distribution, and $f_{\varphi}(\varphi)$ is the p.d.f. of $\varphi$, which can be shown to be $f_{\varphi}(\varphi)=2(n-1)(\sin \varphi)^{2 n-3} \cos \varphi$. Using these, the average BER was evaluated in [7]. It was also shown that the effect of the optimal ordering is a 3 $\mathrm{dB}$ increase in the SNR. However, no explanation was provided for this effect.

In order to get more insight into the algorithm performance and, in particular, into the optimal ordering procedure, we study in this paper the joint CDF 


$$
F\left(x_{1}, x_{2}\right)=\operatorname{Pr}\left[\left|\mathbf{h}_{1 \perp}\right|^{2}<x_{1},\left|\mathbf{h}_{2 \perp}\right|^{2}<x_{2}\right]
$$

and the joint PDF,

$$
\rho\left(x_{1}, x_{2}\right)=\frac{\partial^{2} F\left(x_{1}, x_{2}\right)}{\partial x_{1} \partial x_{2}}
$$

While $\mathbf{h}_{1}$ and $\mathbf{h}_{2}$ are independent (by the assumption of i.i.d. Rayleigh channel), the interference nulling out introduces correlation between $\left|\mathbf{h}_{1 \perp}\right|^{2}$ and $\left|\mathbf{h}_{2 \perp}\right|^{2}$. Using the same argument as in $[6,7]$, the joint CDF can be presented as

$$
F\left(x_{1}, x_{2}\right)=(n-1) \int_{1}^{\infty} F_{n}\left(x_{1} t\right) F_{n}\left(x_{2} t\right) t^{-n} d t
$$

The joint PDF is

$$
\rho\left(x_{1}, x_{2}\right)=(n-1) \int_{1}^{\infty} \rho_{n}\left(x_{1} t\right) \rho_{n}\left(x_{2} t\right) t^{-n+2} d t
$$

where

$$
\rho_{n}(x)=\frac{d F_{n}(x)}{d x}=\frac{x^{n-1}}{(n-1) !} e^{-x}
$$

is the n-th order MRC PDF. After some manipulations, (11) reduces to the following

$$
\rho\left(x_{1}, x_{2}\right)=\frac{n}{(n-2) !}\left(\frac{x_{1} x_{2}}{x_{1}+x_{2}}\right)^{n-1} \frac{e^{-\left(x_{1}+x_{2}\right)}}{\left(x_{1}+x_{2}\right)^{2}} \sum_{i=0}^{n} \frac{\left(x_{1}+x_{2}\right)^{i}}{i !}
$$

Note that, as it should be due to the problem symmetry, the PDF is symmetrical with respect to $x_{1}$ and $x_{2}$. It is straightforward to see that

$$
\rho\left(x_{1}\right)=\int_{0}^{\infty} \rho\left(x_{1}, x_{2}\right) d x_{2}=\frac{x_{1}^{n-2}}{(n-2) !} e^{-x_{1}}
$$

as it should be since $\left|\mathbf{h}_{1 \perp}\right|^{2} \sim \chi_{2(n-1)}^{2}$. Asymptotically, for $x_{1}, x_{2}<<1$ (i.e., small outage probability region), one obtains

$$
\rho\left(x_{1}, x_{2}\right) \approx \frac{n}{(n-2) !}\left(\frac{x_{1} x_{2}}{x_{1}+x_{2}}\right)^{n-1} \frac{1}{\left(x_{1}+x_{2}\right)^{2}}
$$

After some manipulations, (10) can be presented in the following form:

$F\left(x_{1}, x_{2}\right)=1-(n-1)\left(I_{2}\left(x_{1}\right)+I_{2}\left(x_{2}\right)-I_{2}\left(x_{1}+x_{2}\right)-I_{32}\right)$

where

$$
\begin{gathered}
I_{2}(x)=e^{-x} \sum_{i=0}^{n-2} a_{i} x^{i}, a_{i}=(n-i-2) !(-1)^{i} \sum_{j=0}^{i} \frac{(-1)^{j}}{(n-1-j) ! j !} \\
I_{32}=e^{-\left(x_{1}+x_{2}\right)}\left(x_{1}+x_{2}\right)^{n-1} \sum_{i=n}^{2 n-2} \varphi_{i}\left(x_{1}, x_{2}\right)\left(x_{1}+x_{2}\right)^{-i} \\
\varphi_{i}\left(x_{1}, x_{2}\right)=\sum_{j=i}^{2 n-2} \sum_{k=j-n+1}^{n-1} \frac{(j-n) ! x_{1}{ }^{k} x_{2}^{j-k}}{k !(j-k) !(j-i) !}
\end{gathered}
$$

Note that, while $F(x, x)=P(x)$, as it should be, $\rho(x, x) \neq d P(x) / d x$. Asymptotically, for $x_{1}, x_{2}<<1$, the joint $\mathrm{CDF}$ is

$$
F\left(x_{1}, x_{2}\right) \approx \frac{1}{(n-1) !}\left(\frac{x_{1} x_{2}}{x_{1}+x_{2}}\right)^{n-1}
$$

For $x_{1}<x_{2}$, one obtains the marginal CDF,

$$
F\left(x_{1}, x_{2}>>x_{1}\right) \approx F\left(x_{1}\right) \approx \frac{1}{(n-1) !} x_{1}^{n-1}
$$

which is the asymptotic CDF of (n-1)-th order MRC, as it should be since $\left|\mathbf{h}_{1 \perp}\right|^{2} \sim \chi_{2(n-1)}^{2}$. For $x_{1}=x_{2}$, (17) clearly gives the $3 \mathrm{~dB}$ effect first predicted in [6,7].

Using the joint PDF, we can now evaluate the moments of $\left[x_{1}, x_{2}\right]$,

$$
\begin{aligned}
& \overline{x_{1}}=\overline{x_{2}}=(n-1), \sigma_{1}^{2}=\overline{\left(x_{1}-\overline{x_{1}}\right)^{2}}=(n-1), \sigma_{2}^{2}=\sigma_{1}^{2} \\
& \overline{x_{1} x_{2}}=\frac{n^{2}(n-1)}{n+1}, \mathrm{R}_{12}=\frac{\overline{x_{1} x_{2}}-\overline{x_{1}} \cdot \overline{x_{2}}}{\sigma_{1} \sigma_{2}}=\frac{1}{n+1}
\end{aligned}
$$

The expression for the correlation coefficient is remarkably simple. Note that the correlation decreases as $\mathrm{n}$ increases and for large $\mathrm{n}$ it is very small. However, it cannot be neglected as the diversity order analysis in [6,7] demonstrates. For example, in our case $(\mathrm{m}=2)$ neglecting the correlation would result in $s=\max \left[\left|\mathbf{h}_{1 \perp}\right|^{2},\left|\mathbf{h}_{2 \perp}\right|^{2}\right]$ having diversity order $=2(\mathrm{n}-1)$ while, in fact, the true diversity order is (n-1). Let us consider this issue in more details. If we assume that $\left|\mathbf{h}_{1 \perp}\right|^{2}$ and $\left|\mathbf{h}_{2 \perp}\right|^{2}$ are independent, then the CDF of $s$ is

$$
F_{s}^{\prime}(x)=\operatorname{Pr}\{s<x\}=F_{n-1}^{2}(x)
$$

However, the true CDF of $\mathrm{s}$ is given by,

$$
F_{S}(x)=\operatorname{Pr}\{s<x\}=F(x, x)
$$

The results are very different for the small outage probabilities, as fig. 2 demonstrates.

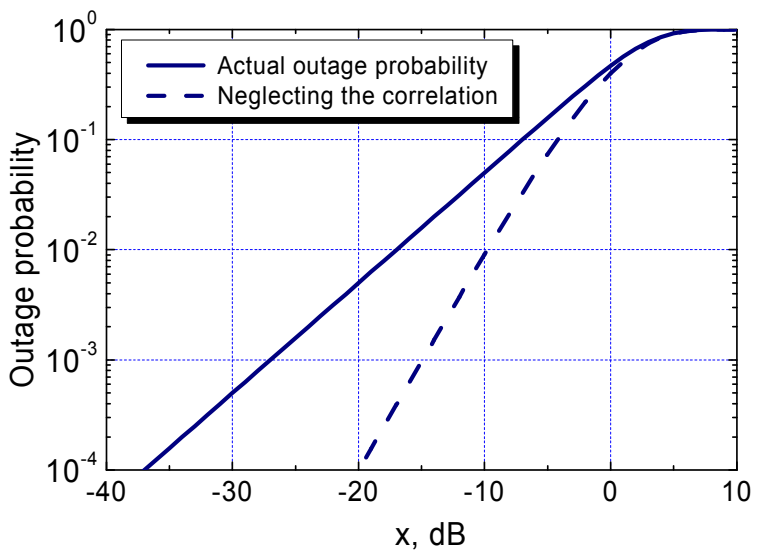

Figure 2. Comparing the true $1^{\text {st }}$ step outage probability with the one when the correlation is neglected, for $2 \times 2$ system.

The reason for low correlation being important is that we are interested in the distribution tails (low outage) and the 
correlation is just an average measure which does not emphasize the tails. It should be also noted that zero correlation does not in general means independence (this is true for the Gaussian random variables but, in our case, $\left|\mathbf{h}_{1 \perp}\right|^{2}$ and $\left|\mathbf{h}_{2 \perp}\right|^{2}$ are not Gaussian).

It is instructive to consider the $2 \times 2$ case in more details, when the expressions become especially simple. One obtains,

$$
\begin{gathered}
\rho\left(x_{1}, x_{2}\right)=2 x_{1} x_{2} e^{-\left(x_{1}+x_{2}\right)} \times \\
\times\left(\frac{1}{\left(x_{1}+x_{2}\right)^{3}}+\frac{1}{\left(x_{1}+x_{2}\right)^{2}}+\frac{1}{2\left(x_{1}+x_{2}\right)}\right) \\
F\left(x_{1}, x_{2}\right)=1-\left(e^{-x_{1}}+e^{-x_{2}}\right)+e^{-\left(x_{1}+x_{2}\right)}\left(1+\frac{x_{1} x_{2}}{x_{1}+x_{2}}\right)
\end{gathered}
$$

The corresponding asymptotic expressions, for $x_{1}, x_{2}<<1$, are

$$
\begin{gathered}
\rho\left(x_{1}, x_{2}\right) \approx \frac{2 x_{1} x_{2}}{\left(x_{1}+x_{2}\right)^{3}} \\
F\left(x_{1}, x_{2}\right) \approx \frac{x_{1} x_{2}}{x_{1}+x_{2}}
\end{gathered}
$$

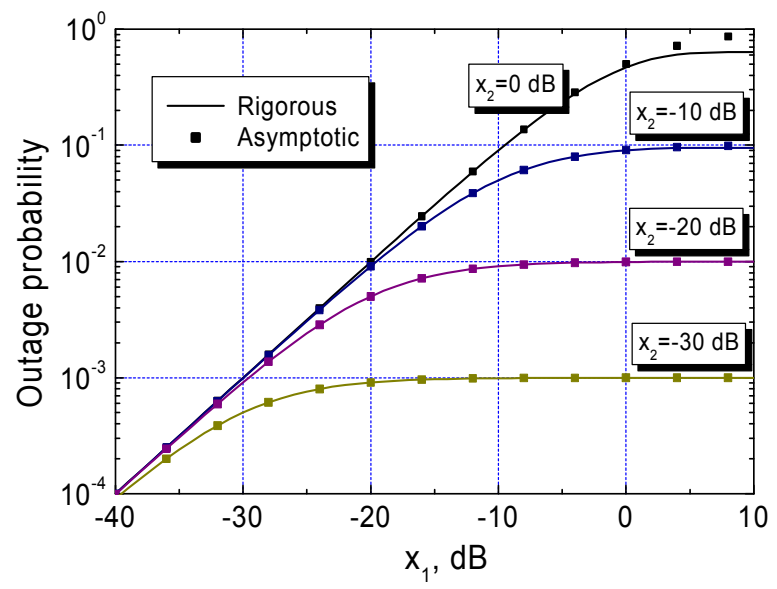

Figure 3. Rigorous and approximate expressions for the outage probability for $2 \times 2$ system.

Fig. 3 compares the asymptotic expression (25) with the rigorous one. As one might see, $(25)$ is very accurate for $x_{1}, x_{2}<1$. Extensive Mote-Carlo simulations have been carried out to verify the analytical expressions. The results are not distinguishable from the analytical one and, hence, are not shown on the figure. Clearly, when $x_{2}<x_{1}$ there is a floor effect: increasing further $x_{1}$ does not decrease the outage probability (as it would be the case if $x_{1}$ and $x_{2}$ were independent). Hence, the joint outage probability is dominated by the smallest signal power. This is the way in which $x_{1}$ and $x_{2}$ are coupled to each other. Fig. 4 clearly indicates this effect in 3-D. Similar results hold true for the larger $\mathrm{n}$ as well.

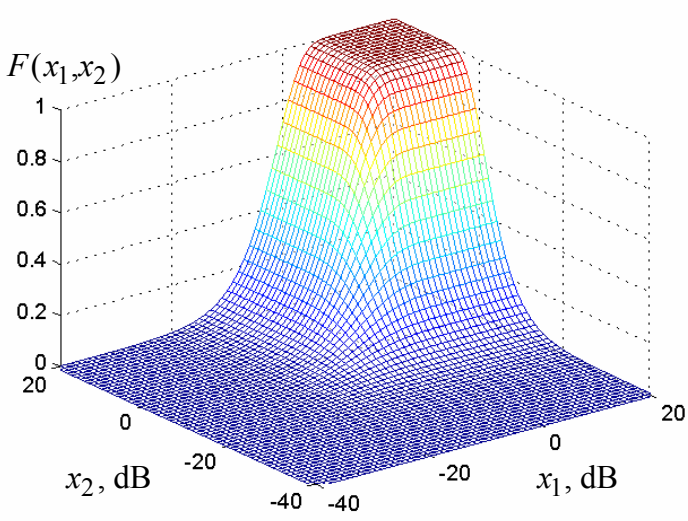

Figure 4. Outage probability versus $x_{1}$ and $x_{2}$.

\section{GENERIC CASE OF MXN SYSTEM}

It is difficult to obtain similar results in the case of $m>2$ since the joint pdf $f_{\varphi}\left(\varphi_{1}, \ldots \varphi_{m}\right)$ of $\left\{\varphi_{1}, \ldots \varphi_{m}\right\}, \varphi_{i}$ being the angle between $\mathbf{h}_{i}$ and the sub-space spanned by all the other column vectors, is not known. However, based on the asymptotic CDF (17), we conjecture that in the generic case of mxn system it takes the following form:

$$
F\left(x_{1} \ldots x_{m}\right) \approx \frac{1}{(n-m+1) !}\left(\sum_{i=1}^{m} \frac{1}{x_{i}}\right)^{-n+m-1}
$$

Extensive Monte-Carlo simulations have been carried out for $3 \times 3,3 \times 4$ systems. It was found that the approximation is indeed correct provided that $x_{1}, \ldots, x_{m}<1$.

\section{REFERENCES}

[1] G.J. Foschini et al, Analysis and Performance of Some Basic Space-Time Architectures, IEEE Journal Selected Areas Comm., v. 21, N. 3, pp. 281-320, April 2003.

[2] G.J Foschini, 'Layered space-time architecture for wireless communication in a fading environment when using multiple antennas', Bell Lab. Tech. J., vol. 1, N. 2, pp. 41-59, 1996.

[3] G.J Foschini et al, Simplified Processing for High Spectral Efficiency Wireless Communication Employing Multi-Element Arrays, IEEE Journal on Selected Areas in Communications, v. 17, N. 11, pp. 1841-1852, Nov. 1999.

[4] M. Schwartz, W.R. Bennett, S. Stein, Communication Systems and Techniques, IEEE Press, New York, 1996.

[5] M.K. Simon, M.S. Alouini, Digital Communication over Fading Channels, Wiley, New York, 2000.

[6] S. Loyka, V-BLAST Outage Probability: Analytical Analysis, IEEE Vehicular Technology Conference, 24-28 September 2002, Vancouver, Canada.

[7] S. Loyka, F. Gagnon, Performance Analysis of the V-BLAST Algorithm: an Analytical Approach, IEEE Trans. Wireless Comm., accepted, 2003. 